\title{
Miller Fisher syndrome diagnosis and treatment in a patient with SARS-CoV-2
}

\author{
Paolo Manganotti ${ }^{1}$ - Valentina Pesavento ${ }^{2}$ - Alex Buoite Stella ${ }^{1} \cdot$ Lucia Bonzi $^{2}$ - Elena Campagnolo ${ }^{3}$ - Giulia Bellavita ${ }^{1}$. \\ Bruno Fabris $^{3} \cdot$ Roberto Luzzati $^{4}$
}

Received: 15 April 2020 / Revised: 12 May 2020 / Accepted: 14 May 2020 / Published online: 11 June 2020

(C) Journal of NeuroVirology, Inc. 2020

\begin{abstract}
This case report describes the clinical characteristics of a 50-year-old woman that developed SARS-CoV-2 pneumonia and was admitted at the COVID-19 dedicated unit where she developed neurological symptoms 10 days after admission. After neurological examination, including a panel of blood cerebrospinal fluid biomarkers, a diagnosis of Miller Fisher syndrome (MFS) was hypothesized and intravenous immunoglobulin therapy (IVIG) was initiated. Fourteen days after the start of IVIG treatment, the patient has been discharged at home with the resolution of respiratory symptoms and only minor hyporeflexia at the lower limbs, without any side effect.
\end{abstract}

Keywords COVID-19 $\cdot$ Miller Fisher syndrome $\cdot$ Cranial polyneuritis $\cdot$ Immunoglobulins

The diffusion of the novel coronavirus SARS-CoV-2 has rapidly spread worldwide, with several cases requiring intensive care and hospitalization because of the severe respiratory symptoms requiring assisting devices. Miller Fisher syndrome (MFS), a variant of Guillain-Barré syndrome (GBS), is characterized by the clinical triad of ophthalmoplegia, ataxia, and areflexia. About $60 \%$ of the above mentioned autoimmune syndromes can be infection-related by humoral and cellular cross-reactivity (Pusch et al. 2018), most frequently gastrointestinal (Campylobacter jejuni) or respiratory tract infections, including influenza (Sellers et al. 2017).

Paolo Manganotti

pmanganotti@units.it

1 Clinical Unit of Neurology, Department of Medicine, Surgery and Health Sciences, Cattinara University Hospital ASUGI, University of Trieste, Strada di Fiume, 447, 34149 Trieste, Italy

2 Rehabilitation Unit, Cattinara University Hospital ASUGI, Via Giuseppe Lorenzo Gatteri, 25/1, 34125 Trieste, Italy

3 Endocrinology Unit, Department of Medicine, Surgery and Health Sciences, Cattinara University Hospital ASUGI, University of Trieste, Strada di Fiume, 447, 34149 Trieste, Italy

4 Infectious Diseases Department, Department of Medicine, Surgery and Health Sciences, Cattinara University Hospital ASUGI, University of Trieste, Strada di Fiume, 447, 34149 Trieste, Italy
A previously healthy 50 -year-old female, who worked as a healthcare assistant in an assisted living community, was admitted to the hospital with a diagnosis of bilateral pneumonia as a consequence of the SARS-CoV-2 infection. The first symptoms 6 days before the admission were fever and cough, and a significant alteration of taste was reported. In the COVID-19 protected area, she was treated with antiviral therapy (lopinavir + ritonavir) for 14 days, hydroxychloroquine for 10 days, antibiotic therapy, and oxygen support (35\%). Ten days after hospital admission, the pulmonary function improved, but the patient developed neurological signs such as diplopia and facial paresthesia. The first neurological examination found walking impairment due to ataxia, ophthalmoplegia with diplopia in vertical and lateral gaze, left upper arm cerebellar dysmetria, generalized areflexia, mild lower facial defects, and mild hypoesthesia in the left mandibular and maxillary branch of the face.

To exclude a posterior circulation stroke, a magnetic resonance imaging (MRI) of the brain was performed, which revealed no abnormalities. The results of routine blood chemistry tests, anti-HIV, anti-HBV, and anti-HCV, and a panel of serological tests of autoimmune disorders were unremarkable. The cerebrospinal fluid (CSF) analysis revealed clear CSF, normal pressure, and no blood cells. The CSF/serum glucose ratio was $80 / 110 \mathrm{mg} / \mathrm{dL}$. CSF protein concentration was $74.9 \mathrm{mg} / \mathrm{dL}$, higher compared with normal values $<45 \mathrm{mg} /$ dL. CSF culture and polymerase chain reaction (PCR) for 
possible organisms, such as bacteria, Mycobacterium tuberculosis, fungi, Herpes viruses, Enteroviruses, Japanese $B$ virus, and Dengue viruses, yielded negative results.

Neurophysiological evaluation, as electroneuromyography, was not possible because of the limitations due to the COVID19 protected area. A panel of AGAbs, including anti-GM1, anti-GM2, anti-GM3, anti-GD1a, anti-GD1b, anti-GT1b, and anti-GQ1b, was negative.

Based on the clinical presentation and CFS findings, an intravenous immunoglobulin (IVIG) therapy was initiated at $0.4 \mathrm{~g} / \mathrm{kg}$ for 5 days. The neurological symptoms resolved 7 days after the start of IVIG treatment, with complete recovery of diplopia and dysmetria, and the patient was able to walk without signs of ataxia. After the acute phase, the patient remained in the COVID-19 protected area still needing respiratory support. No side effects were reported for the use of intravenous immunoglobulin therapy. Fourteen days after the start of IVIG treatment, the patient has been discharged at home with the resolution of respiratory symptoms and only minor hyporeflexia at the lower limbs.

\section{Discussion}

The clinical presentation of the reported case, and CSF analysis showing a picture of albumin-cytological dissociation, suggested the diagnosis of MFS as previously described in the literature (Wakerley et al. 2014). The novelty of this case is represented by the diagnosis of MFS in a COVID-19 patient and by the clinical suggestion of treating neurological complications with intravenous immunoglobulin therapy. Such neurological complications are common in respiratory infections (Sellers et al. 2017); therefore, a cross-reactivity also for the new SARS-CoV-2 was speculated and reported (Zhao et al. 2020) as for SARS-CoV affected patients (Baig et al. 2020). We did not find any presence of anti-GQ1b, usually explaining the symptoms of the disease (Wakerley et al. 2014). However, negative results for anti-GQ1b tests have been previously reported (Wattanasit and Sathirapanya 2020). The particular cranial polyradiculoneuritis with the involvement of the facial and trigeminal nerve is well-known in MFS and MFS variants (Polo et al. 1992; Wakerley et al. 2014), and in the reported case, it was found being associated to an altered sense of taste, which is an uncommon feature of MFS but well-reported in COVID-19. IVIG was found to be effective and safe to treat the reported neurological symptoms, showing complete recovery after 7 days. In conclusion, this case report describes the characteristics of a MFS/cranial polyneuritis in a patient with COVID-19, and the clinical responses to intravenous immunoglobulin therapy, suggesting possible diagnosis and treatment options in this peculiar condition.

Acknowledgments The authors want to thank all the physicians, nurses, and staff of the COVID-19 protected area at ASUGI.

\section{Compliance with ethical standards}

Conflict of interest The authors declare that they have no conflicts of interests.

\section{References}

Baig AM, Khaleeq A, Ali U, Syeda H (2020) Evidence of the COVID-19 virus targeting the CNS: tissue distribution, host-virus interaction, and proposed neurotropic mechanisms. ACS Chem Neurosci 11: 995-998. https://doi.org/10.1021/acschemneuro.0c00122

Polo A, Manganotti P, Zanette G, De Grandis D (1992) Polyneuritis cranialis: clinical and electrophysiological findings. J Neurol Neurosurg Psychiatry 55:398-400. https://doi.org/10.1136/jnnp. 55.5.398

Pusch E, Renz H, Skevaki C (2018) Respiratory virus-induced heterologous immunity: part of the problem or part of the solution? Allergo J Int 27:79-96. https://doi.org/10.1007/s40629-018-0056-0

Sellers SA, Hagan RS, Hayden FG, Fischer WA 2nd (2017) The hidden burden of influenza: a review of the extra-pulmonary complications of influenza infection. Influenza Other Respir Viruses 11:372-393. https://doi.org/10.1111/irv.12470

Wakerley BR, Uncini A, Yuki N (2014) Guillain-Barré and Miller Fisher syndromes-new diagnostic classification. Nat Rev Neurol 10:537544. https://doi.org/10.1038/nrneurol.2014.138

Wattanasit P, Sathirapanya P (2020) Anti-ganglioside antibody-negative Miller Fisher and AMSAN variant Guillain-Barré overlap syndrome. Case Rep Neurol 12:92-96

Zhao H, Shen D, Zhou H, Liu J, Chen S (2020) Guillain-Barré syndrome associated with SARS-CoV-2 infection: causality or coincidence? Lancet Neurol 19:383-384. https://doi.org/10.1016/S14744422(20)30109-5

Publisher's note Springer Nature remains neutral with regard to jurisdictional claims in published maps and institutional affiliations. 